'Departamento de Bioética y Humanidades Médicas, Facultad de Medicina, Universidad de Chile.Santiago, Chile. apsicóloga. ${ }^{b} \mathrm{PhD}$.

Trabajo no recibió financiamiento. Los autores declaran no tener conflictos de interés.

Recibido el 28 de enero de 2020, aceptado el 26 de enero de 2021.

Correspondencia a:

M. Luz Bascuñán R. Departamento de Bioética y Humanidades Médicas, Facultad de Medicina, Universidad de Chile.

Avenida Independencia 2027 Santiago, Chile. mlbascunan@gmail.com

\section{Problemas Éticos en la Práctica Médica. Estudio exploratorio desde la perspectiva de médicos de dos hospitales públicos}

\author{
M. LUZ BASCUÑÁN R. ${ }^{1, \mathrm{a}, \mathrm{b}}$, SERGIO VALENZUELA P. ${ }^{1}$
}

\section{Perspectives of physicians who work in public hospitals about ethical problems in medical practice}

Background: The recognition of ethical problems in medical practice and the potential harmful effects that they may have on patients, underscores the importance of the ethical training of professionals as well as the development of Ethics Committees in Healthcare institutions. However, there is a paucity of national studies about the type of problems faced by professionals and the role of Ethics Committees in hospitals. Aim: To explore the perception about ethical problems in clinical practice of Chilean physicians of different specialties of two public teaching hospitals. Material and Methods: Physicians of both sexes and different specialties working in two teaching hospitals of Metropolitan Santiago, were invited to participate in focus groups of seven to eight participants. They discussed the ethical problems faced during their clinical practice. A content analysis based on the Grounded Theory was performed with the obtained information. Results: Different types of ethical problems could be distinguished according to their content and the actors involved. The data obtained shows that the ethical dimension is present in the daily work of physicians. The evolution of the narrative throughout the interviews corroborated the importance of installing ethical issues and culture in the working routine of health care professionals. Conclusions: Human resources and an adequate institutional environment are required to promote the discussion about ethical issues such as the relationship between physicians and patient, the role of teaching in health care and institutional decisions.

(Rev Med Chile 2021; 149: 458-463)

Key words: Consultants; Ethics, Clinical; Hospitals.
L a literatura sobre los problemas éticos en medicina frecuentemente proviene de una reflexión conceptual entre expertos. Se alude a los desafíos que nos plantean los avances científico-tecnológicos y la creciente participación de los usuarios en la atención de salud. También los cambios socio-culturales y legales como aquellos en el sistema de salud y la medicina, han generado nuevos desafíos teniendo un impacto más bien negativo en los médicos ${ }^{1,2}$.

Los estudios empíricos sobre la frecuencia y tipo de problemas éticos en medicina, utilizan distintas aproximaciones metodológicas dificultando su comparación. De hecho, estas dificultades comenzarían con la misma definición de problema ético ${ }^{3}$. 
Sin embargo, dos estudios han sido paradigmáticos en el tema: el de DuVal, Clarridge, Gensler, Danis (2004) en Estados Unidos ${ }^{4}$ y el de Hurst, Perrier, Pegoraro, Reiter-Theil, Forde, Slowther, Garrett-Mayer y Danis (2007) en Europa ${ }^{5}$. Ambos estudios fueron realizados con el objetivo de orientar los servicios de ayuda ética. Mientras el primero enfatiza las variaciones entre las sub-especialidades médicas, el segundo recalca la importancia de realizar estudios "situados" en un sistema de salud y contexto socio-cultural particular.

En el estudio de DuVal et al. (2004) los problemas éticos más frecuentemente reportados por los médicos se asocian a decisiones en el final de la vida, autonomía del paciente, justicia y conflictos relacionales. Se encontró diferencias respecto a las variables sub-especialidad y formación ética. Mientras los internistas reportaron más dilemas asociados a la justicia, los oncólogos lo hicieron respecto a la comunicación de la verdad. Otros estudios confirman que médicos de distintas especialidades varían en la frecuencia y tipo de problemas éticos ${ }^{6,7}$. Adicionalmente, los profesionales con mayor formación ética identifican problemas y utilizan asesorías éticas más frecuentemente.

Por su parte Hurst et al. (2007) aplicaron el cuestionario de DuVal et al. a médicos internistas europeos. Las dificultades éticas experimentadas se vincularon a la capacidad para la toma de decisiones del paciente, desacuerdo entre profesionales o cuidadores y limitación de tratamiento. El nivel de dificultad atribuida por los médicos a los problemas varió significativamente entre países y esto se atribuye a diferencias culturales en las nociones de salud, enfermedad y muerte. Además, los mismos sistemas de salud y comités de consultorías éticas presentan características particulares.

Si bien en Chile actualmente la mayoría las instituciones de salud cuentan con Comités de Ética Asistencial (CEAs), se han realizado escasos esfuerzos por explorar la calidad de su funcionamiento y aporte a la institución ${ }^{8}$.

Ramos y Bedregal (2017) identificaron los principales temas de ética médica en la práctica clínica de acuerdo a una revisión de los artículos publicados en revistas de Medicina y Bioética chilenas entre 1998 y 2013. Encontraron que los "deberes y obligaciones" deontológicos es el tema central de la discusión en ética médica tiñendo los demás temas incluyendo: relación médicopaciente, objeción de conciencia, autonomía del paciente, entre otros. Este estudio corrobora la falta de investigación empírica en este tema y en especial la carencia de estudios que den cuenta de la perspectiva de los propios médicos 9 .

El objetivo de este estudio es explorar la percepción que sostiene una muestra de médicos de distintas especialidades de dos hospitales públicos docente-asistenciales de la región metropolitana, respecto a los problemas éticos que experimentan en su práctica clínica y las estrategias que se utilizan para su enfrentamiento.

\section{Material y Método}

Se realiza un estudio de tipo exploratorio-descriptivo 9 que utiliza una aproximación metodológica cualitativa consistente con el interés de explorar un área para la cual carecemos de información desde la perspectiva de los mismos actores sociales involucrados ${ }^{10}$.

\section{Instrumento}

Como método de recolección de datos se llevaron a cabo grupos focales permitiendo generar información proveniente de diversas personas simultáneamente ${ }^{11}$. Éstos se realizaron en base a un guión de preguntas estructuradas. Siguiendo los criterios tradicionales, se realizó dos grupos focales en tanto se consideró adecuado el nivel de saturación de la información ${ }^{12}$.

\section{Participantes}

Los grupos focales se realizaron en dos hospitales públicos docentes de la Región Metropolitana y estuvieron integrados por 8 y 7 participantes respectivamente. En ambas ocasiones hubo médicos hombres y mujeres, de distintas especialidades (ginecología, cirugía, neurología, intensivista, radiología, inmunología, cardiología, psiquiatría, hematología). Se excluyó de la muestra a quienes son miembros de un comité de ética.

Este estudio contó con la aprobación de los Comité de Ética de la Investigación de los dos hospitales en que se llevó a cabo el estudio.

\section{Procedimiento de análisis de datos}

Se realizó un análisis de contenido basado en la "Teoría fundamentada" cuya característica central es la fundamentación y emergencia de conceptos y teorías basada en los datos empíricos. A través 
de un proceso inductivo se distinguen y analizan categorías y subcategorías conceptuales ${ }^{13}$. En primer lugar, se realiza una codificación abierta por dos investigadores independientemente. Así se identificó unidades de significado y se construyó un esquema de clasificación jerárquica. A partir de este esquema se hizo un análisis descriptivo de la información y luego un análisis relacional, vinculando conceptualmente las diferentes categorías temáticas.

\section{Resultados}

Con el objetivo de ilustrar las categorías temáticas identificadas se utiliza algunas citas de los participantes.

\section{Frecuencia en que se experimentan problemas éticos}

Las opiniones respecto a la frecuencia con que se experimentan problemas éticos se asocian a la noción de problema ético, a la especialidad y varían a lo largo de las entrevistas. En un principio resulta difícil identificar problemas éticos y éstos tienden a ser trasgresiones a normas y principios, pero luego se comienzan a referir conflictos en los roles docente y clínico, respecto a pacientes, colegas, residentes y autoridades. En la unidad de cuidado crítico, a diferencia de las otras especialidades, se experimentaría problemas éticos asociados a decisiones del fin de vida constantemente.

\section{Tipos de Problemas}

\section{Relación médico-paciente}

La primera categoría se relaciona a todas aquellas fallas y dificultades en la atención del paciente de acuerdo a las mínimas buenas prácticas clínicas lo que supone un trato comprometido y personalizado con el paciente.

Por lo tanto, casos de descoordinación de las unidades asistenciales o en los que falta un médico referente para el paciente atentan contra la buena práctica. Es decir, "hay un problema al parcelar y diluir las responsabilidades, pedir interconsultas y no hay un médico que unifique las opiniones de las distintas especialidades".

Por su parte, se cuestiona éticamente que no se ofrezca la información necesaria al paciente y sus familiares, y que se actúe sin consultarle ni explicarle su situación de salud.

\section{Voluntad del paciente}

Una serie de conflictos tiene relación con los derechos y la participación del paciente en la toma de decisiones. Esto incluye problemas asociados al debido proceso de un correcto consentimiento informado.

También se cuestiona "hasta donde la voluntad del paciente tiene peso sobre la indicación o decisión técnica" ya sea rechazando o solicitando un tratamiento. Se alude a la negativa del paciente a ser atendido por un estudiante (en un hospital docente) y se critican los casos en que el residente tendría un rol central para el paciente en tanto "responde a las necesidades no satisfechas por parte del médico".

\section{Carácter docente de la Institución}

Por una parte, se cuestiona que se delegue en estudiantes tareas asistenciales complejas para las que no están preparados. Por otra parte, se critica que no se integre a los estudiantes en la actividad clínica y que los médicos se nieguen a hacer docencia. En ambos casos se identifica un "conflicto de interés donde el beneficio del médico y del servicio es priorizado por sobre el interés del paciente".

\section{Recursos y normas institucionales}

Para algunos médicos la falta de recursos del medio hospitalario constituye un problema ético significativo. "Una de las peores cosas es tener que decirle al paciente "hasta aquí llegamos" por que el sistema no financia su tratamiento. Uno se siente muy mal".

Se critican ciertas políticas institucionales y se atribuye una serie de problemas a la proporción de actividades docente-asistenciales y a los horarios parciales. Se cuestiona severamente el uso de la institución para fines personales. Se alude a la falta de tiempo para atender adecuadamente al paciente y su familia; hacer docencia; colaborar con los colegas y sentirse parte de la institución.

Existiría una cultura institucional conformada por las prácticas habituales y valoraciones implícitas. Así muchas veces las "conductas del médico "poco éticas" son parte de la cultura del hospital".

\section{Enfrentamiento de Problemas éticos}

Normas y compromiso: Se considera que muchos problemas éticos surgen por la carencia 
de normas y por tanto se supone que el establecimiento de reglas, su socialización y el velar por su cumplimiento reduciría los problemas éticos.

La importancia atribuida a la falta de regulaciones tiende a referir la solución a estos problemas a la gestión administrativa. "Porque aquí hay alguien que tiene que tener autoridad y que tiene que estar controlando, tiene que haber sanción también, eso hace que funcione el sistema”.

Es necesario el compromiso de los médicos con las normas y las autoridades. Tal compromiso iría de la mano de la participación de los profesionales en los lineamientos de la institución. "Hay que normar y hacer valer las normas. Pero además tiene que haber participación, que nos sintamos pertenecientes a esta institución".

Escala de ayuda: Si bien se debe tomar decisiones con implicaciones éticas constantemente, en general éstas se toman espontáneamente sin necesidad de asesoría. Se conoce y valora el CEA, pero se posterga la solicitud de ayuda porque "necesitas decisiones clínicas muy rápidas" y la asesoría del comité demora.

El comité de ética asistencial sería un recurso al que se acude en última instancia, sólo cuando el problema ético no ha podido resolverse entre colegas o con la dirección del servicio. "Uno va al comité si no hay acuerdo entre el grupo". "Es la segunda etapa, lo vemos primero localmente”.

\section{Discusión}

Los problemas éticos referidos en este estudio tienden a apelar a los valores de la responsabilidad y compromiso del médico con los pacientes, los colegas, los equipos de salud y la institución. Consistente con la literatura internacional se mencionan problemas en la relación clínica, vinculados a la autonomía del paciente, y se distingue conflictos éticos asociados al fin de la vida propios de la unidad de cuidado crítico $^{4,5}$.

No obstante, el carácter docente-asistencial de las instituciones en este estudio, presenta un conjunto de dificultades éticas adicionales a nivel profesional e institucional incluyendo el grado de participación de los estudiantes en la atención clínica, la reacción del paciente a ser tratados por un médico en formación, el balance (desbalance) y coordinación (descoordinación) entre las actividades docentes y asistenciales y entre actividades de estudiantes y médicos docentes. La narración de los participantes muestra gran variedad de intereses y presiones que los médicos deben conciliar y resistir, así como un conjunto de exigencias a los cuadros directivos.

La evolución del discurso de los médicos a lo largo de las entrevistas grupales muestra la importancia y necesidad de generar espacios de discusión de estos temas en los equipos de trabajo. La reflexión conjunta permitió ir precisando problemas éticos de distinto tipo y a diferentes niveles, así como reconocer cierto nivel de malestar moral ${ }^{14,15}$.

Más allá de la motivación y la sensibilidad moral personal, son las autoridades de las organizaciones y las jefaturas de equipo los llamados a asumir su responsabilidad en instalar e incorporar estos temas a la reflexión habitual en las dinámicas de trabajo.

En este estudio se observó que las decisiones ante un conflicto ético suelen tomarse intuitiva y aisladamente. En casos más complejos se cuenta primero con los colegas, luego directivos y por último el comité de ética asistencial del hospital.

Si bien se distinguen problemas éticos derivados del manejo personal de situaciones y de las políticas institucionales; éstos no son independientes. El enfrentamiento de los dilemas de "gestión personal" frecuentemente depende de la cultura hospitalaria. La ética institucional facilitaría y normalizaría las prácticas de grupos e individuos.

En efecto, sabemos que la resiliencia moral, antídoto del malestar moral, requiere de un "ambiente o cultura ética” en la institución en la que se trabaja ${ }^{16,17}$.

En esta línea se sostiene que los problemas éticos se reducirían mediante el establecimiento de normas claras y la facilitación de un ambiente institucional comprometido y participativo.

Si bien la existencia de normas y directrices pueden contribuir a orientar los cursos de acción y a unificar criterios para la toma de decisiones, éstas no eliminan las fuentes de conflicto ni sustituye el necesario proceso de deliberación para el enfrentamiento de cada caso. En esta línea cabe apelar a la tarea educativa y propositiva (no sólo resolutiva) que poseen los Comités de Ética asistencial en las instituciones de las que forman parte.

Este trabajo sólo exploró la perspectiva de los médicos sobre los problemas éticos en la práctica clínica. La relevancia de una cultura ética institucional en la que sus miembros compartan un 
discurso ético deliberativo cotidiano; requiere de futuros estudios que examinen el tema en otros profesionales y trabajadores de los equipos de salud así como en el personal administrativo y directivo.

\section{Conclusiones}

La práctica médica contemporánea está marcada por el progresivo y irreversible desplazamiento de los actos profesionales, que se dan dentro de una relación médico-paciente a la de un entramado de relaciones y decisiones que complejizan la acción médica. Este escenario, ya descrito suficientemente, no solo la hacen posible, sino que además suele hacer mas segura, mas accesible y colaborativa la medicina. Esto por supuesto que tensiona la percepción de los médicos sobre hasta donde puede llegar su quehacer autónomo frente al paciente individual y cuanto depende de la institución, de los "otros" (pagadores, interdisciplinas, roles docente-científico-gestor-interconsultor-prestador-etc).

En relación al rol que juega la ética en este nuevo escenario. P. Simón ha destacado hace tiempo la necesidad de dar pasos decisivos hacia un nuevo estadio de la ética clínica, la de la ética de las organizaciones sanitaria. En este punto es donde los autores creemos que se intersectan muy bien, los hallazgos del presente estudio exploratorio. Hay una percepción de ello en casi todas las reclamaciones que se realizan transversalmente entre las diferentes especialidades médicas. El nuevo escenario de la acreditación de las instituciones de salud, ponen de manifiesto, la necesidad de dar respuesta a las exigencias crecientes a las que se sienten sometidos los profesionales sanitarios. Nuevos roles y nuevos escenarios clínicos.

La vía de resolución de los conflictos percibidos, al parecer, no pueden ser resueltos (al menos totalmente) ni en forma individual por los profesionales ni por las estructuras actuales de los comité de ética asistenciales, los reglamentos y normas, las guias y las vías clínicas. Se requiere, a nuestro juicio, simultáneamente, de procesos planificados de formación en ética para los profesionales, de instancias colaborativas y colegiadas de reflexión sobre las prácticas profesionales y de liderazgos fuertes que conduzcan estos procesos de mejora continua y de calidad en las organizaciones.
Agradecimientos: Agradecemos a todos los médicos que participaron generosamente en este estudio compartiendo sus opiniones y experiencias.

\section{Referencias}

1. Jiménez JP, Horwitz N, Bascuñán ML, Acuña J, Fullerton C. "Las transformaciones de la medicina y su impacto en la calidad de la vida de los médicos". Documento de trabajo No9. Corporación de Promoción Universitaria (CPU), 2003.

2. Bascuñán ML. Cambios en la relación médico-paciente y nivel de satisfacción de los médicos. Rev Med Chile 2005; 133 (1): 11-6.

3. Braunack Mayer AJ. What makes a problem an ethical problem? An empirical perspective on the nature of ethical problems in general practice. J Med Ethics 2001; 27: 98-103.

4. DuVal G, Clarridge B, Gensler G, Danis M. A national survey of U.S. internists' experiences with ethical dilemmas and ethics consultation. J Gen Intern Med. 2004; 19: 251-8.

5. Hurst SA, Perrier A, Pegoraro R, Reiter-Theil S, Forde $\mathrm{R}$, Slowther AM, et al. Ethical difficulties in clinical practice: experiences of European doctors. J Med Ethics 2007; 33: 51-7.

6. Oerlemans A, Sluisveld N, Leeuwen E, Wollersheim H, Dekkers W, Zegers M. Ethical problems in intensive care unit admission and discharge decisions: a qualitative study among physicians and nurses in the Netherlands. BMC Med Ethics 2015; 16: 9.

7. Saarni SI, Halila R, Palmu P, Vänska J. Ethically problematic treatment decisions in different medical specialties. J. Med Ethics 2008; 34: 262-7.

8. Valenzuela S. Comité de Ética Asistencial Hospital Clínico de la Universidad de Chile: memoria de 10 años de trabajo. Rev. Hosp. Clin Univ Chile 2018; 29: 223-8.

9. Ramos P, Bedregal P. La ética médica en discusión: análisis de los principales temas sobre ética médica en la práctica clínica tratados en revistas nacionales de medicina y bioética 1998-2013. Acta Bioethica 2017; 23 (1): 71-81.

10. Creswell JW. Research Design. Qualitative, Quantitative and Mixed Method Approaches. (2 edición). Londres: SAGE, 2009.

11. Tomat C. El 'focus group': nuevo potencial de aplicación en el estudio de la acústica urbana. Athenea Digital 2012; 12 (2): 129-52.

12. Hyman K. The Focus Group Design. Dept. Of Sociology, SUNY at Stony Brook. New York, 1986.

13. Corbin J y Strauss A. Basics of Qualitative Research: 
Problemas Éticos en medicina - M. L. Bascuñán et al

Techniques and Procedures for Developing Grounded Theory ( $3^{\text {a }}$ ed.). California, EE.UU.: Sage, 2008.

14. Fourie C. Moral distress and moral conflict in clinical ethics. Bioethics 2015, 22 (2): 91-7.

15. Austin CL, Saylor R, Finley P. Moral distress in physicians and nurses: Impact on professional quality of life and turnover. Psychological Trauma: Theory, Research, Practice, and Policy 2017; 9 (4): 399-406.

16. Rushton CH. Cultivating Moral Resilience. Am J Nurs. 2017; 117: 11-5.

17. Stutze K y Bylone M. Building moral resilience. Critical Care Nurse 1918; 38 (1): 77-9.

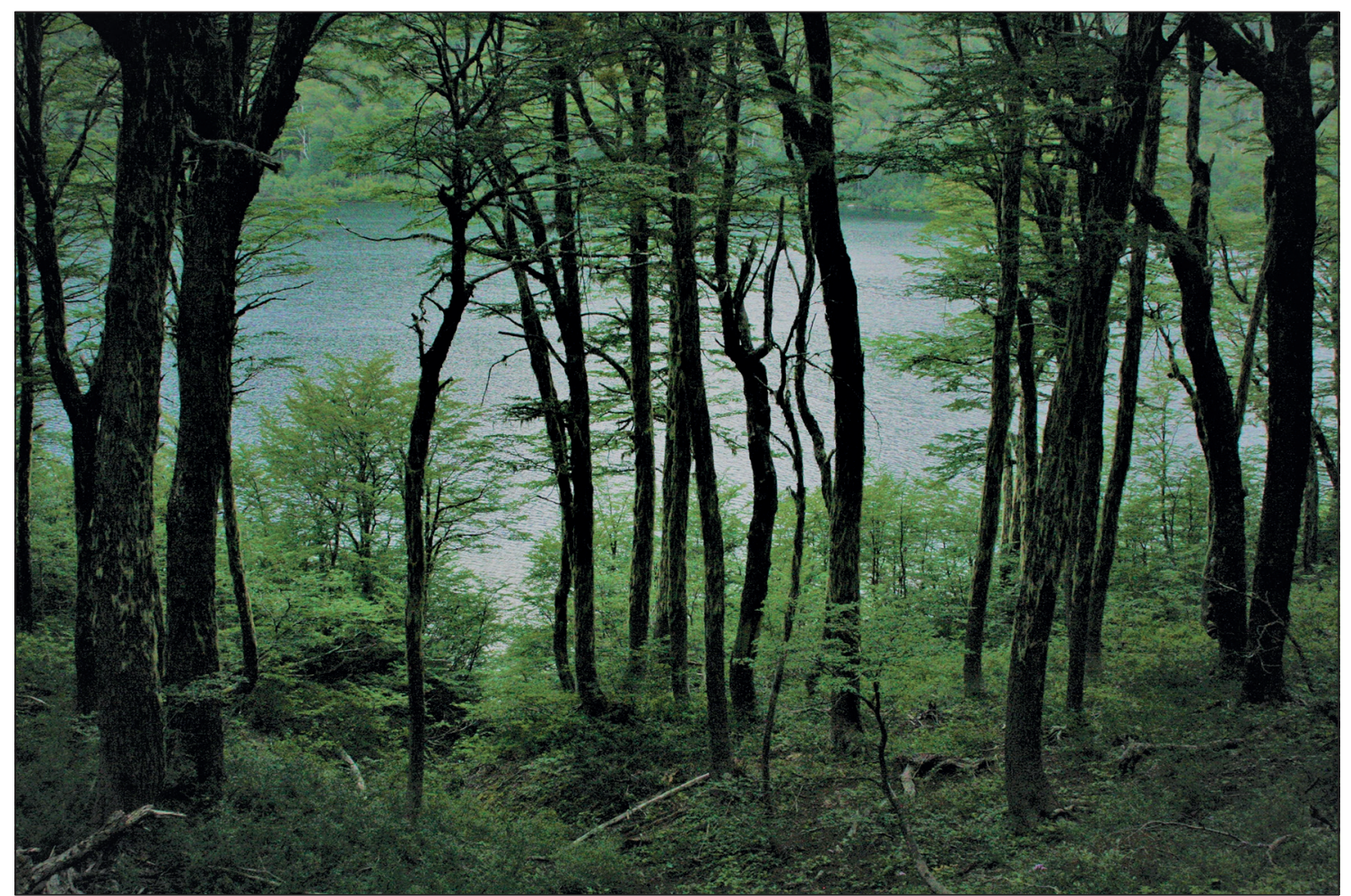

Sendero Laguna Avutardas, Curarrehue, La Araucanía. Dr. Jorge Sapunar Zenteno 